\title{
TRADITIONAL CHINESE MEDICINE
}

\section{Protective effect of glycyrrhizin on nephrotic syndrome induced by adriamycin in rats}

Li Yu${ }^{1}$

XueCheng Bi PhD'2

Gang Zhu ${ }^{4}$

ZhaoDong Han ${ }^{1}$

YongKang Ye ${ }^{1}$

YuXiang Liang ${ }^{1}$

Lei Zhang ${ }^{1}$

ZhiHong Hao ${ }^{1}$

GuoHua Zeng ${ }^{3}$

HuiChan $\mathrm{He}^{1}$

WeiDe Zhong1,2
${ }^{1}$ Guangzhou First Municipal People's Hospital, Af-

filiated Guangzhou Medical College, Guangzhou

510180, China

${ }^{2}$ Southern Medical University, Guangzhou 510180, China

${ }^{3}$ The First Affiliated Hospital of Guangzhou Medical

College, Guangzhou 510182, China

${ }^{4}$ Beijing Hospital MH, Beijing 100730, China

Manuscript submitted 4th February, 2009

Manuscript accepted 24th April, 2009

Clin Invest Med 2009; 32 (3): E229-E238.

\begin{abstract}
Purpose: To explore the protective effect of glycyrrhizin in rats with nephrotic syndrome (NS) induced by adriamycin (ADR).

Methods: 36 Sprague Dawley (SD) male rats were divided into control, untreated and glycyrrhizin treatment groups. The NS rat model was established by injecting ADR twice in the untreated and in the glycyrrhizin treatment groups. Rats in the glycyrrhizin treatment group were fed glycyrrhizin by intragastric administration for 7 days. Changes in the following indices were observed in the three groups before and 4 weeks after the treatment: $24 \mathrm{~h}$ urine protein quantitation (UPr), serum cholesterol (Ch), serum albumin (Alb), blood urea nitrogen (BUN), serum creatinine ( $\mathrm{sCr}$ ), laminin (LN), fibronectin (FN), collagen (Col), transforming growth factor $\beta 1$ (TGF $\beta 1$ ) and connective tissue growth factor (CTGF); histopathology by light and electron microscope. Expression of LN, FN, ColIV, TGF $\beta 1$ and CTGF in the cortex of the kidney were detected by semiquantitive immunohistochemical analysis. Expression of TGF $\beta 1$ and CTGF in the cortex of the kidney was detected by Fluorescein Based Quantitive RT-PCR. Macrophage infiltration was evaluated by the immunoperoxidase staining.
\end{abstract}

(C) 2009 CIM
Results: Compared with the control group, 24h UPr, Ch, $\mathrm{BUN}$ and $\mathrm{sCr}$ of rats in the untreated group were increased. Glycyrrhizin reduced 24h Upr, Ch, BUN, sCr, LN, FN, Col, TGF $\beta 1$, CTGF, and mean arterial blood pressure. Pathological changes in the kidney, the expression of $\mathrm{LN}$, FN, Col, TGF $\beta 1$ and CTGF in the cortex of the kidney in the glycyrrhizin treatment group were decreased compared with the untreated group. Glycyrrhizin also suppressed macrophage infiltration in the kidneys of NS rat models.

Conclusion: Glycyrrhizin exerts protective effects in rats with NS, reducing the excretion of $\mathrm{Upr}, \mathrm{Ch}, \mathrm{BUN}, \mathrm{sCr}$, and mean arterial blood pressure, and also decreasing expression of LN, FN, Col, TGF $\beta 1$ and CTGF in the kidney. Renal function is improved and the severity of NS is lessened.

Nephrotic syndromes (NS) may develop in many mammalian species as a result of primary diseases such as minimal change disease in humans, inflammatory diseases such as membranous nephropathy or immune glomerulonephritis in humans or laboratory animals, or exposure to toxic substances such as adriamycin (ADR) in rats. ${ }^{1}$ Besides proteinuria and

Clin Invest Med • Vol 32, no 3, June 2009 
podocyte foot process effacement, the development of edema and ascites are permanent clinical symptoms in the majority of sufferers ${ }^{2}$ The pathogenesis of NS is unclear. Some reports have indicated that NS can lead to glomerular sclerosis and renal failure. Mesangial cell proliferation and extracellular matrix (ECM) accumulation are the main pathological features of NS. Transforming growth factor $\beta 1$ (TGF $\beta 1$ ) and connective tissue growth factor (CTGF) participate in the process of glomerular disease and ECM accumulation, which may result in glomerular sclerosis ${ }^{3}$ Inhibition of cytokines related to glomerular sclerosis and ECM accumulation is the key factor in preventing glomerular sclerosis.

For thousands of years, many herbs have been used as natural remedies for the prevention and/or treatment of renal diseases. Various herbs and herbal products are believed to have kidney protective functions and are widely used in clinical practice in the West as well as in the East. Glycyrrhizin, the major active component extracted from licorice (Glycyrrhiza glabra) roots, is one of the oldest and most commonly prescribed herbs in Eastern traditional medicine, and has been used to treat tuberculosis, peptic ulcers, and liver injury in a number of clinical disorders in human patients. ${ }^{4,5}$ Glycyrrhizin may scavenge reactive oxygen species (ROS) and has an anti-inflammatory action.6,7 Recent publications have reported that glycyrrhizin could down-regulate expression of transcription factor $\kappa \mathrm{B}, \mathrm{CTGF}$ and TGF $\beta 1$ in rats with obstructive nephropathy, and inhibit the occurrence of renal interstitial fibrosis. ${ }^{8}$

ADR causes NS in rats that corresponds to minimal change nephropathy or focal segmental glomerulosclerosis. ${ }^{9-10}$

In the present study, we produced rat models of NS by intravenous injection of ADR. Rats in the glycyrrhizin treatment group were fed glycyrrhizin for 7 days. The following indices were observed before and 4 weeks after the treatment: urine protein quantitation (UPr) of 24h, serum cholesterol (Ch), serum albumin (Alb), blood urea nitrogen (BUN), serum creatinine $(\mathrm{sCr})$, laminin $(\mathrm{LN})$, fibronectin $(\mathrm{FN})$, collagen IV(CollV), Transforming Growth Factor $\beta 1$ (TGF $\beta 1$ ), Connective Tissue Growth Factor (CTGF) and the mean arterial blood pressure. Histopathological changes were detected by light and electron microscopy on the cortex of the kidney tissues. Expression of LN, FN and $\mathrm{Col}$ in the renal cortex was detected by immunohistochemistry. Expression of TGF $\beta 1$ and CTGF in the kidney tissues was detected by fluorimetry based on quantitive RT-PCR. Finally, the changes of these markers were compared among the three groups to determine the protective effect of glycyrrhizin in rats with NS.

\section{Materials and Methods}

\section{Experimental animals}

The study group consisted of 36 adult male SpragueDawley (SD) rats weighing 240-260g, which were obtained from the Department of Laboratory Animal Science, Guangzhou Medical School, Guangdong province of P.R.China. All animals were treated humanely and the Medical Laboratory Animal Management Committee of Guangzhou Medical School approved all animal procedures. The rats were housed in a temperature controlled room $\left(24 \pm 1^{\circ} \mathrm{C}\right)$ on a 12 hour light and dark cycle, with free access to food and water.

\section{Materials}

Glycyrrhizin (Stronger Neo Minophagen C, \#H20030184) was purchased from Jian'an Medicine Ltd of Shenzhen, Guangdong province, P.R.China. Adriamycin (doxorubicin HCL, \#0408E1, ADR) was supplied by Wanle Medicine Ltd of Shenzhen, Guangdong province, P.R.China. Rabbit anti-LN, -FN, -Col polyclonal antibodies and Streptavidinbiotin peroxidase complex immunohistochemical assay kit (SABC) were obtained from Wuhan BOSTER Ltd., Hubei province, P.R.China. Metabolic cages 
(\#3700M071) were bought from Tecniplast Gazzade S.ar.1 Ltd.

\section{Preparation of animal models and drug treatment}

$36 \mathrm{SD}$ rats were randomly divided into three groups: control, untreated and glycyrrhizin treatment groups, with 12 rats in each group. NS was induced by a single dose of ADR $(5 \mathrm{mg} / \mathrm{kg})$ injected into the tail vein of conscious rats in the untreated model and the glycyrrhizin treatment groups twice, once every 7 days. After modeling, rats in the glycyrrhizin treatment group were fed with glycyrrhizin for 7 days $(200 \mathrm{mg} / \mathrm{kg}$ glycyrrhizin by intragastric administration), whereas rats in the control and the untreated groups were fed normal saline solution for 7 days. Twelve rats of each group were chosen for biochemical measurement, while 10 rats were selected for the morphological evaluation. The survival rate of the glycyrrhizin treatment group was $93.86 \%$.

\section{Quantitative detection of $24 \mathrm{~h}$ UPr and blood biochemical detection}

24h UPr (urinary protein in $\mathrm{mg} / \mathrm{ml}$ glomerular filtrate in $24 \mathrm{~h}$ ) was determined by spectrophotometry, after $3 \%$ sulfosalicylic acid precipitation of urine collected from rats individually housed in metabolic cages for $24 \mathrm{~h}$ before ADR administration and 4 weeks after drug treatment. Blood samples ( $3 \mathrm{ml}$ from inguinal vein) were collected and centrifuged to separate the plasma. The biochemical parameters ( $\mathrm{sCh}, \mathrm{Alb}, \mathrm{BUN}$, sCr, LN, FN, Col, TGF $\beta 1$ and CTGF) levels in the plasma were measured according to the users' instruction of diagnostic kits (Roche AG, Switzerland) and by the biochemical analyzer Cobas Mira plus (Roche AG, Switzerland) in Guangzhou Medical School, Guangdong province of P.R.China. The parameters were detected by OLYMPUS AU2700 Automatic Biochemical Analyzer. Blood pressure was monitored continuously until $60 \mathrm{~min}$ after the administration of glycyrrhizin and stored.

\section{Histopathological Detection}

Four weeks after drug treatment, rats were anaesthetized with $10 \%$ chloral hydrate $(0.5 \mathrm{ml} / 100 \mathrm{~g})$ by ip injection. One kidney was removed and fixed in 10\% formalin, embedded in paraffin and examined in multiple consequent sections. Histopathologic study was carried out using PAS staining and observed by light microscopy. Additionally, other partial kidney tissues were fixed by $3 \%$ glutaraldehyde and $1 \%$ osmic acid in order, and stained by uranyl acetate and citric acid. Then, the histopathological changes in the renal cortex were observed by electron microscopy. To evaluate glomerular size, 100 glomeruli/group were examined. Glomerular surface area $\left(\mu \mathrm{m}^{2}\right)$ was determined in digital images by a single blinded observer using the Soft Imaging System (Olympus, London, UK).

\section{Immunohistochemistry Assay}

Expression of LN, FN, Col, TGF $\beta 1$ and CTGF in kidney tissue was analyzed by immunohistochemical staining. Tissues were fixed in $10 \%$ buffered formalin and embedded in paraffin. Commercially available monoclonal antibodies to LN, FN, Col, TGF $\beta 1$ and CTGF were used. Immunohistochemical staining was carried out using the avidin biotin method and a commercially available kit. One paraffin-embedded block of kidney tissue was selected from each case and cut into $4 \mu \mathrm{m}$ sections. Deparaffinized sections were treated with methanol containing 3\% hydrogen peroxide for $10 \mathrm{~min}$ before conducting antigen retrieval using a microwave oven at $95^{\circ} \mathrm{C}$ for 5 minutes and cooling at $25^{\circ} \mathrm{C}$ for $2 \mathrm{hr}$. After washing with phosphate buffered saline (PBS), blocking serum was applied for $10 \mathrm{~min}$. The sections were incubated with an anti LN monoclonal antibody (1:300), anti FN monoclonal antibody $(1: 300)$, anti Col monoclonal antibody (1:50), anti TGF $\beta 1$ monoclonal antibody (1:500) and anti CTGF monoclonal antibody (1:25) overnight at $4^{\circ} \mathrm{C}$. Negative control sections were incubated with PBS instead of the primary antibody. After washing in PBS, a biotin marked secondary anti- 
body was applied for 10 min followed by a peroxidase marked streptavidin for an additional $10 \mathrm{~min}$. The reaction was visualized using 3, 3'-diaminobenzidinetetrahydrochloride. The nuclei were counterstained with hematoxylin. Positive and negative immunohistochemistry controls were routinely used. Reproducibility of staining was confirmed by reimmunostaining via the same method in multiple, randomly selected specimens. Two experienced pathologists, blinded to the description of the slices, examined the stained specimens independently.

Images were collected by Olympus DD70 BX51 (Olympus, Japan) and analyzed by IMAGE-PRO plus 4.1 software (Media Cybernetics, USA). Ten visual fields in each section were randomly selected and the value of the relative optical density (OD) was measured. Five to ten representative replicate sections were assessed per animal and a minimum of 6 animals were analyzed per group. The extent of immunohistochemical staining of LN, FN, Col, TGF $\beta 1$ and CTGF was semi-quantified by the mean value of OD in every section.

\section{Fluorimeter Based Quantitive RT-PCR Assay}

The mRNA expression of TGF $\beta 1$ and CTGF in rat kidney tissue in each group was detected by fluorescein based quantitive RT-PCR. Total RNA was extracted from the cortex of the kidney with a phenol/ guanidine isothiocyanate based reagent (TrizolTM, Gibco BRL, Scotland.). Briefly, $1 \mathrm{ml}$ Trizol and 200 $\mu 1$ chloroform were added to each tube. The RNA was then precipitated with $500 \mu \mathrm{l}$ isopropanol $(\mathrm{v} / \mathrm{v})$ and washed with $75 \%$ ethanol and air dried. The purified RNA was then dissolved in $10 \mu$ diethyl pyrocarbonate (DEPC) treated water. The optical density was measured at $260 / 280$ wavelength and stored at $-80^{\circ} \mathrm{C}$ prior to being used in the synthesis of cDNA.

RNA was reverse transcribed using the Super scripte $^{\mathrm{TM}}$ Preamplification System (Life Technology). 500ng total RNA were mixed with 500ng oligo (dT) primers and incubated at $70^{\circ} \mathrm{C}$ for $10 \mathrm{~min}$. The mix- ture was then chilled on ice and incubated with a $1 \times$ reverse transcriptase buffer $(50 \mathrm{mM}$ Tris- $\mathrm{HCl}, \mathrm{pH}$ $8.4,75 \mathrm{mM} \mathrm{KCl}), 3 \mathrm{mM} \mathrm{MgCl}, 500 \mu \mathrm{M}$ of each deoxynucleotide, $10 \mathrm{mM}$ dithiothreitol and $200 \mathrm{U}$ of $\mathrm{Su}-$ perscript II RT reverse transcriptase at $42^{\circ} \mathrm{C}$ for 50 $\mathrm{min}$. The $20 \mu \mathrm{l}$ reactions were further incubated at $70^{\circ} \mathrm{C}$ for $15 \mathrm{~min}$ and $2 \mathrm{U}$ RNase $\mathrm{H}$ was added to each tube. Following final incubation at $37^{\circ} \mathrm{C}$ for $20 \mathrm{~min}$, the cDNAs were stored at $-80^{\circ} \mathrm{C}$ until being used.

PCR was performed in MJ Opticon Monitor 2.0 (MJ Ltd, USA) using SYBR Green I (Biogene) as fluorescein. The following pairs of primers were used: $\beta$-actin (258bp): 5'GAC CTT CAA CAC CCC AGC CA 3' (sense), 5' GTC ACG CAC GAT TTC CCT CTC 3' (antisense);' TGF $\beta 1$ (209bp): 5' GTG GAC CGC AAC AAC GCA 3'(sense), 5' ACC AAG GTA ACG CCA GGA AT 3'(antisense); CTGF (133bp) 5' GCG TAA AGC CAG GGA GTA 3' (sense); 5' AGC AGT TAG GAA CCC AGA TT 3' (antisense). All primers were synthesized by Sangon Co. (Shanghai, China). The PCR profile consisted of an initial melting step of $2 \mathrm{~min}$ at $94^{\circ} \mathrm{C}$, followed by 39 cycles of 45 $\mathrm{s}$ at $94^{\circ} \mathrm{C}, 20 \mathrm{~s}$ at $62^{\circ} \mathrm{C}$ and $20 \mathrm{~s}$ at $72^{\circ} \mathrm{C}$, and a final elongation step of $10 \mathrm{~min}$ at $62^{\circ} \mathrm{C}$.

Sample quantification was carried out with Sequence Detection System (SDS2.0) software constructing a standard calibration curve using serial dilutions of $\beta$-actin DNA of known concentration, from which the concentration of an unknown sample could be determined. In order to analyze interassay variation, a least square curve fitting test was used to construct a fit, and the standard error of the data points to the curve was estimated by plotting the log of the sample concentration against the number of cycles the PCR machine undertook to yield a set of fluorescent data. The standard error was estimated to be $15 \%$ on the $\log$ and was within the values described for $\beta$-actin quantification using competitive PCR. 
TABLE 1. Quantitative results of $24 \mathrm{hUPr}, \mathrm{sCh}, \mathrm{Alb}, \mathrm{BUN}, \mathrm{sCr}$ in each group $(\bar{x} \pm s)$

\begin{tabular}{l|c|c|c|c|c|c}
\hline Group & $24 \mathrm{UPr}(\mathrm{mg} / 24 \mathrm{~h})$ & $\mathrm{Alb}(\mathrm{g} / \mathrm{L})$ & $\mathrm{sCh}(\mathrm{mmol} / \mathrm{L})$ & $\mathrm{sCr}(\mu \mathrm{mol} / \mathrm{L})$ & $\mathrm{BUN}(\mathrm{mmol} / \mathrm{L})$ & $\mathrm{Blood}$ pressure $(\mathrm{mmHg})$ \\
\hline Control & $16.7 \pm 3.8$ & $34.2 \pm 3.5$ & $1.7 \pm 0.2$ & $33.6 \pm 5.0$ & $4.7 \pm 1.0$ & $126.2 \pm 9.3$ \\
\hline Untreated & $60.6 \pm 4.7^{2}$ & $20.5 \pm 1.6^{2}$ & $4.0 \pm 0.8^{2}$ & $75.1 \pm 6.6^{2}$ & $11.8 \pm 1.6^{2}$ & $132.8 \pm 10.1^{1}$ \\
\hline Glycyrrhizin & $46.4 \pm 8.3^{2,4}$ & $28.1 \pm 2.9^{23}$ & $2.3 \pm 0.9^{2,3}$ & $52.6 \pm 9.0^{1,3}$ & $8.8 \pm 1.1^{1,3}$ & $127 \pm 5 \pm 9.6$ \\
\hline
\end{tabular}

All results were expressed as mean $\pm \mathrm{SD}$. (n=12)

${ }^{1} P<0.05,{ }^{2} P<0.01$ vs. the control group, ${ }^{3} P<0.05,{ }^{4} P<0.01$ vs. the untreated model group.

TABLE 2. Quantitative changes in levels of LN, FN, ColIV, TGF $\beta 1$ and CTGF in blood plasma in different groups $(\bar{x} \pm s)$

\begin{tabular}{l|c|c|c|c|c}
\hline Group & $\mathrm{LN}(\mu \mathrm{g} / \mathrm{L})$ & $\mathrm{FN}(\mu \mathrm{g} / \mathrm{L})$ & ColIV $(\mu \mathrm{g} / \mathrm{L})$ & TGF $\beta 1(\mathrm{ng} / \mathrm{L})$ & CTGF $(\mathrm{ng} / \mathrm{L})$ \\
\hline Control & $42.5 \pm 7.5$ & $81.5 \pm 3.2$ & $23.7 \pm 12.7$ & $22.7 \pm 10.5$ & $18.6 \pm 14.7$ \\
\hline Untreated & $83.6 \pm 9.0^{\mathbf{1}}$ & $196.9 \pm 2.1^{\mathbf{2}}$ & $58.2 \pm 14.8^{2}$ & $46.6 \pm 11.3^{2}$ & $40.6 \pm 15.0^{\mathbf{2}}$ \\
\hline Glycyrrhizin & $64.4 \pm 8.4^{\mathbf{2}, 4}$ & $134.3^{2} \pm 4.1^{\mathbf{2}, 4}$ & $42.5 \pm 13.5^{\mathbf{2}, 3}$ & $\left.33.3 \pm 10.2^{\mathbf{2} 3}\right)$ & $30.5 \pm 15.5^{\mathbf{2}, 3}$ \\
\hline
\end{tabular}

All results were expressed as mean \pm SD. $(\mathrm{n}=12)$

${ }^{1} P<0.05,{ }^{2} P<0.01$ vs. the control group, ${ }^{3} P<0.05,{ }^{4} P<0.01$ vs. the untreated model group.

\section{Immunoperoxidase Staining of Macrophages}

To evaluate the infiltration of macrophages, immunoperoxidase staining using an $\mathrm{ABC}$ kit (Vector Laboratories, Burlingame, CA) was performed. Briefly, fresh-frozen kidney sections were used. Nonspecific binding was blocked by incubation with $10 \%$ normal sheep serum in Tris-buffered saline for $20 \mathrm{~min}$ to reduce background interference. Nonspecific staining was blocked by 15 -min incubation with avidin and then biotin using an avidin-biotin blocking kit (Vector Laboratories). Endogenous peroxidase activity was inhibited by 20 min incubation with methanol containing $0.3 \% \mathrm{H}_{2} \mathrm{O}_{2}$. Sections were first incubated with a monoclonal antibody against rat monocytes/ macrophages (ED1) for $12 \mathrm{~h}$ at $4^{\circ} \mathrm{C}$. The sections were then incubated with biotin-labeled goat antimouse IgG for $30 \mathrm{~min}$. Biotinylated horseradish peroxidase was applied for $30 \mathrm{~min}$. Peroxidase activity was developed in 3, 3-diaminobendine. Mayer's hematoxylin was added as a counterstain.

Intraglomerular ED1-positive cells were counted in 200 glomeruli/group by two independent observers with no prior knowledge of the experimental design. The average number per glomerulus was used for the estimation.

\section{Calculations and statistics}

Data obtained from the above experiments were expressed as mean \pm S.D. $(\bar{x} \pm s)$ and handled by SPSS12.0 software. Data was analyzed by one way ANOVA with the post hoc Tukey's test applied for paired comparisons and Kruskal Wallis for non parametric test. A difference was considered significant if the $P<0.05$.

\section{Results}

Quantitative Detection of $24 \mathrm{~h} U \mathrm{Pr}$, Biochemistry and Arterial Blood Pressure

Four weeks after the ADR administration, compared with control, levels of 24UPr, sCh, sCr, BUN, LN, FN, CollV, TGF $\beta 1$, and CTGF in the untreated group were increased while the level of Alb in plasma had decreased (Tables 1 and 2).

Seven 7 days of glycyrrhizin treatment protected the rats from NS by reducing levels of 24UPr, sCh, $\mathrm{sCr}$ and BUN while increasing Alb. The levels of LN, FN, Col, TGF $\beta 1$ and CTGF in the rats' plasma also decreased after glycyrrhizin administration. NS rats developed an increase in mean arterial blood pressure which was reduced by Glycyrrhizin treatment (Table 1). 

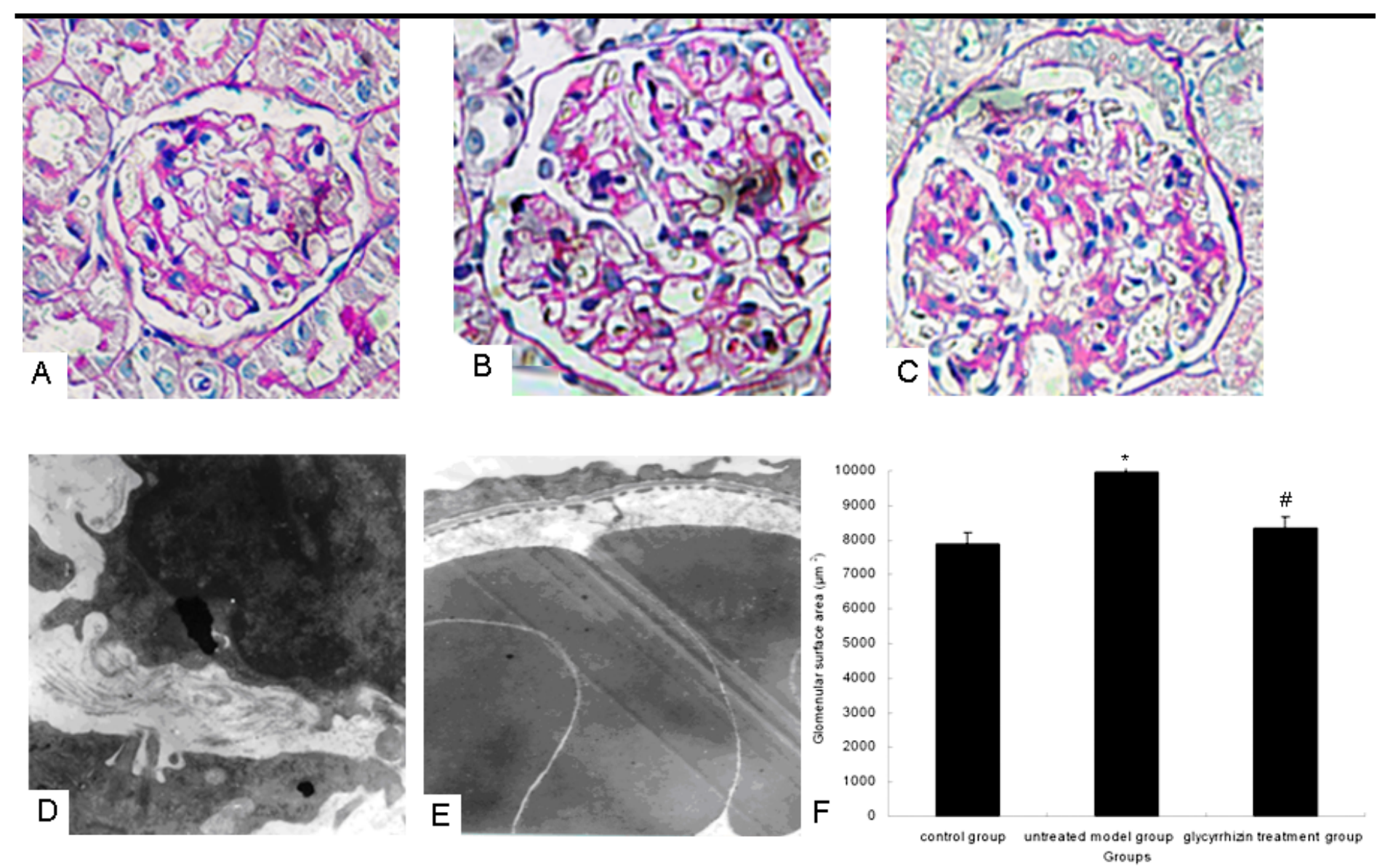

FIGURE 1. Histopathologic changes of kidney tissues in rats of different groups observed by light microscope and electron. A-C: Control group, untreated group and glycyrrhizin treatment group - light microscope; D-E: Untreated group and glycyrrhizin treatment group -electron microscope; Measurement of glomerular surface size in three groups.

\section{Histopathologic Changes in Renal Cortex}

Light microscope photographs show, focal areas of mesangial cell proliferation and vacuolar degeneration, and tubulointerstitial inflammation in kidney tissues in the untreated model group of rats at the time of sacrifice (Figure1A-C). The number of tubulointerstitial cells in the untreated group was greater than in the other two groups. Histopathological changes of mesangial proliferation and tubulointerstitial inflammation were reduced in rats who received glycyrrhizin treatment as were the number of tubulointerstitial cells. In the untreated group, 2 weeks after modeling, inflammatory cell infiltration appeared in the renal interstitium, renal tubules had atrophied a little and the vessel walls were thickened. Four weeks after modeling, these changes were more severe and fibrous degeneration had occurred in the renal interstitium. Furthermore, electron microscopy in untreated rats showed proliferation of intercapillary cells, increase of ECM and collagen fiber-like filaments, and fusion of foot processes (Figure1D-E). After glycyrrhizin, mesangial cell proliferation, foot process confluence, and basement membrane thickening decreased compared with that in the untreated group.

Glomeruli from untreated NS animals tended to be larger than those from normal controls, whereas glomerular surface area was decreased by glycyrrhizin compared with the untreated rats (Figure 1F). Collectively, these results demonstrate that glycyrrhizin decreased glomerular hypertrophy in NS animals. 

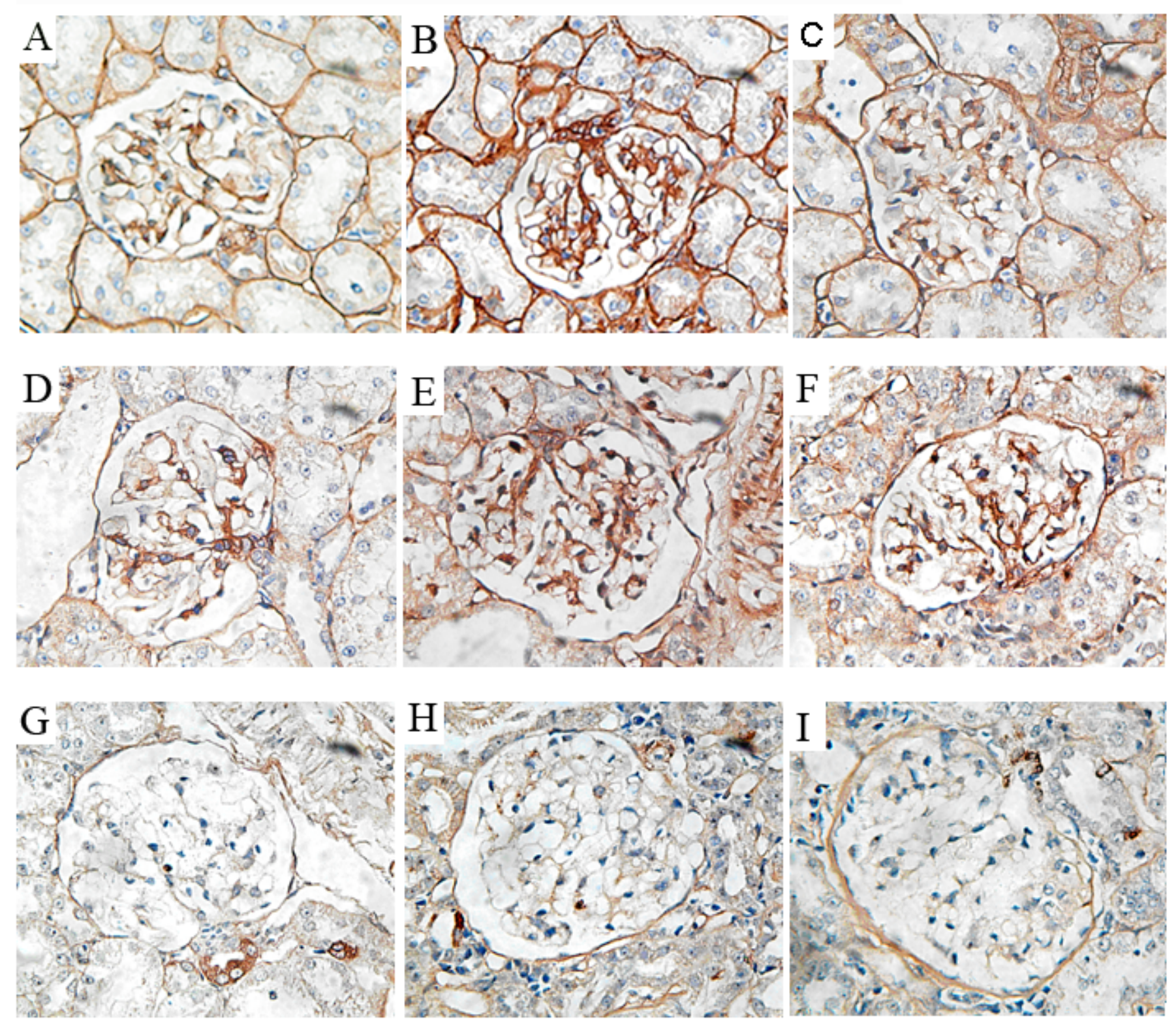

FIGURE 1. Expression of LN, FN and Col in kidney tissue. A-C: LN expression in control group, untreated group and glycyrrhizin treatment group; D-F: FN expression in control group, untreated group and glycyrrhizin treatment group; G-I: Col expression in control group, untreated group and glycyrrhizin treatment group.

Expression of LN, FN, Col, TGFß1 and CTGF Protein in Renal Cortex

There was no expression in the kidneys of animals in the control group (Figure 2A, D and G). In rats with ADR-induced NS, LN, FN and Col were expressed strongly along with the aggravation of NS. Glomcruli showed sclerosis and were occupied completely with
LN, FN and Col (Figure2B, E and H). After glycyrrhizin treatment, expression of LN, FN and Col was weakly positive (Figure 2C, F and I).

$\mathrm{LN}, \mathrm{FN}, \mathrm{Col}, \mathrm{TGF} \beta 1$ and CTGF expression in rats with ADR induced NS was increased compared with normal rats. After glycyrrhizin treatment, expression 
TABLE 3. Semi- quantitative analysis of the expression levels of LN, FN, CollV, TGF $\beta 1$ and CTGF in kidney sections ( $\mathrm{n}=12, \bar{x} \pm s$ )

\begin{tabular}{l|c|c|c}
\hline Markers & Control group & Untreated group & Glycyrrhizin group \\
\hline LN & $1.88 \pm 0.16$ & $2.90 \pm 0.18^{1}$ & $2.21 \pm 0.18^{\mathbf{3}}$ \\
\hline FN & $1.98 \pm 0.14$ & $3.27 \pm 0.23^{1}$ & $2.38 \pm 0.30^{4}$ \\
\hline Col IV & $0.24 \pm 0.04$ & $0.42 \pm 0.06^{2}$ & $0.35 \pm 0.04^{3}$ \\
\hline TGF $\beta 1$ & $1.20 \pm 0.08$ & $3.10 \pm 0.17^{\mathbf{2}}$ & $2.32 \pm 0.13^{\mathbf{3}}$ \\
\hline
\end{tabular}

${ }^{1} P<0.05$ vs. the control group; ${ }^{2} P<0.01$ vs. the control group;

${ }^{3} P<0.05$ vs. the untreated model group; ${ }^{4} P<0.01$ vs. the untreated model group.

TABLE 4. Expression levels of TGF $\beta 1$ and CTGF mRNA in kidney tissue of rats in different groups $(\bar{x} \pm s, \mathrm{ng} / \mathrm{ml}, \mathrm{n}=10)$

\begin{tabular}{l|c|c|c}
\hline & Control group & Untreated model group & Glycyrrhizin treatment group \\
\hline TGF $\beta 1$ & $3.7 \pm 0.8$ & $17.2 \pm 1.4^{\mathbf{2}}$ & $5.1 \pm 0.3^{\mathbf{2 , 3}}$ \\
\hline CTGF & $0.4 \pm 0.1$ & $4.1 \pm 0.3^{\mathbf{2}}$ & $1.9 \pm 0.2^{\mathbf{1 , 3}}$ \\
\hline
\end{tabular}

All results were expressed as mean \pm SD. (ng/ml, $\mathrm{n}=10)$

${ }^{1} P<0.05,{ }^{2} P<0.01$ referred to comparison with the control group, ${ }^{3} P<0.01$ referred to comparison with the untreated model group.

of these markers was lower than in untreated model rats (Table 3).

\section{Expression of TGF $\beta 1$ and CTGF $m R N A$ in Kidney Tissue}

The expression of TGF $\beta 1$ and CTGF mRNA in the untreated group were 4.6 and 10.7 fold higher than in the control group, while the increase in TGF $\beta 1$ and CTGF mRNA were suppressed by glycyrrhizin (Table 4).

\section{Glycyrrhizin Treatment Reduces Glomerular Macrophage Infiltration}

The number of macrophages (ED1-positive cells) in glomeruli was higher in the untreated group than in the control group. Glycyrrhizin treatment reduced the number of glomerular infiltrating macrophages (Figure 3$)$.

\section{Discussion}

In this study, 24UPr, sCh, Alb, BUN, sCr and the mean arterial blood pressure were increased in the rats with NS. In the group receiving glycyrrhizin treatment, these makers were decreased and renal function was ameliorated. Proteinuria effects cellular infiltration. ${ }^{11}$ Protein overload may induce functional alterations of tubular cells, overexpressing proinflammatory mediators. Thus, tubulointerstitial inflammation in NS models corresponds to the time of overt proteinuria, as in this study.

Glycyrrhizin is a glycoside of glycyrrhetinic acid with two molecules of glucuronic acid. It is one of the active ingredients of licorice, roots of Glycyrrhiza glabra and G. ularensis. It has been prescribed fre-

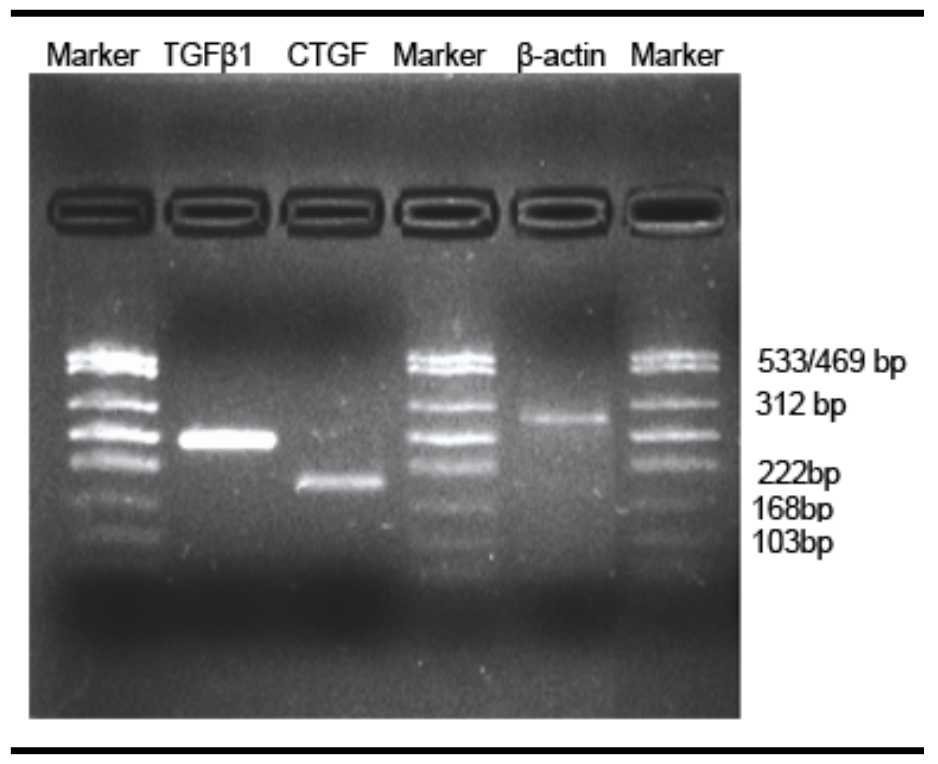

FIGURE 3. The number of macrophages (ED1-positive cells) in glomeruli of NS model rats, normal controls and markedly reduced by glycyrrhizin.

${ }^{\#} P<0.01$; vs. control group, ${ }^{*} P<0.05,{ }^{*} P<<0.01$ 
quently in a herbal Chinese medicine formula to treat various diseases, such as chronic hepatitis $\mathrm{C}$ by decreasing in serum alkaline aminotransferase levels in patients ${ }^{12}$, kidney ischemia-reperfusion injury in animal models ${ }^{13}$ and allergic diseases ${ }^{14}$ because of its anti-inflammatory and antioxidant activities. However, excessive glycyrrhizin may induce peripheral edema, hypokalemia, myopathy, and hypertension, which are symptoms of pseudoaldosteronism. It is potentially fatal. ${ }^{15,16}$ The frequency of pseudoaldosteronism caused by glycyrrhizin depends on the dose and the duration of treatment and is thought to be due to inhibition of type $211 \beta$-hydroxysteroid dehydrogenase $(11 \beta-H S D 2)$ in renal tubular epithelial cells by glycyrrhetic acid. ${ }^{7}$

ADR, an anticancer drug, forms a complex with DNA, inhibiting synthesis of both DNA and RNA. ${ }^{18}$ Kidney injury induced by ADR in a range of laboratory animals is an analogue of NS in humans, the appearances of which are minimal change nephropathy, focal and segmental glomerulosclerosis. ${ }^{19}$ Our histopathological findings showed tubulointerstitial changes with cellular infiltration and vacuolar degeneration in the untreated group, by light and electron microscopy. Interstitial inflammation is considered to be an important determinant of the outcome of glomerular inflammation. Several studies suggested that myofibroblasts of the interstitium may play a crucial role in the pathogenesis of fibrosis in glomerular diseases. $^{20,21}$ Thus, the tubulointerstitial cellular response and vacuolar degeneration seen in our rat model with NS emphasizes the severity of the NS. On the other hand, attenuation of interstitial inflammation and other renal injuries are secondary to inhibition of proteinuria by glycyrrhizin. Glycyrrhizin may delay progression of NS.

Immunohistochemical staining and Fluorimeter based quantitive RT-PCR showed changes in expression of LN, FN, Col, TGF $\beta 1$ and CTGF in different groups. We interpreted this as due to ADR administration and glycyrrhizin treatment. ECM accumulation, caused by disequilibrium of composition and degradation, is considered a principal pathological feature of NS and may lead to glomerular sclerosis, the key factor in the development of NS. ${ }^{22} \mathrm{LN}, \mathrm{FN}$ and ColIV are the ingredients of ECM, while TGF $\beta 1$ and CTGF are important participants in the process of ECM accumulation. ${ }^{23,} 24 \mathrm{We}$ also found that glycyrrhizin suppressed the infiltration of macrophages in the kidneys of NS rats. Therefore, we believe that glycyrrhizin may inhibit ECM accumulation, prevent glomerular sclerosis and relieve the renal injury o NS.

In conclusion, glycyrrhizin exerts protective effects on ADR-induced NS in rats. Glycyrrhizin reduces proteinuria, 24UPr, sCh, $\mathrm{Alb}, \mathrm{BUN}, \mathrm{sCr}$, and the mean arterial blood pressure. Also, it decreases expression of LN, FN, Col, TGF $\beta 1$ and CTGF in kidney tissues, improves renal function and reduces the severity of glomerulosclerosis and retards the development of NS.

\section{Acknowledgments}

This work was supported by grants from the Natural Science Foundation of Guangdong Province (No. 04003650), the Key Programs of Science and Technology of Guangzhou city (No. 200323-E4053), National High Technology Research and Development Project of China (No. 2006AA02A245) and National Natural Science Foundation of China (No. 30872960).

\section{References}

1. Hendrik Bos, Gozewijn D Laverman, Robert H Henning, et al. Involvement of renal ACE activity in proteinuriaassociated renal damage in untreated and treated adriamycin nephrotic rats. J Renin Angiotensin Aldosterone Syst 2003; 4:106-12

2. Bruno Vogt, Bernhard Dick, Hans peter Marti, Felix J Frey, Brigitte M Frey. Reduced $11 \beta$-hydroxysteroiddehydrogenase acticity in experimental nephritic syndrome. Nephrol Dial Transplant 2002; 17:753-8

3. Tomas Zima, Vladimir Tesar, Jirina Crkovska. ICRF 187 (dexrazoxan) protects from adriamycin induced nephritic syndrome in rats. Nephrol Dial Transplant 1998; 13:1975 -9 
4. Rubin Wang, John Kong, Dali Wang, Lin min, Eric Jung. A survey of Chinese herbal ingredients with liver protection activities. Chin Med_2007; 2: 5-13

5. Sohn EJ, Kang DG, Lee HS. Protective effects of glycyrrhizin on gentamicin induced acute renal failure in rats. Pharmacol Toxicol 2003; 93: 116-22

6. Gumpricht E, Dahl R, Devereaux MW, Sokol RJ. Licorice Compounds Glycyrrhizin and 18-Glycyrrhetinic Acid Are Potent Modulators of Bile Acid-induced Cytotoxicity in Rat Hepatocytes. J Biol Chem 2005; 280: 10556-63

7. Yoshida T, Tsuda Y, Takeuchi D, Kobayashi M, Pollard RB, Suzuki F. Glycyrrhizin inhibits neutrophilassociated generation of alternatively activated macrophages. Cytokine. 2006; 33:317-22.

8. Tamaki K, Okuda S, Ando T. TGF $\beta 1$ in glomerulosclerosis and interstitial fibrosis of adriamycin nephropathy. Kidney Int 1994; 45:525-32.

9. Remuzzi G, Zoja C, Remuzzi A. Low protein diet prevents glomerular damage in adriamycin treated rats. Kidney Int 1985; 28: 21-7

10. Deschenes G, Doucet A. Collecting duct ATPase activity is correlated with urinary sodium excretion in rat nephritic syndromes. J Am Soc Nephrol 2000;11:604-15

11. Seza Ozen, Yusuf Usta, Inci Sahin Erdemli, Dicle Orhan. Association of nitric oxide production and apoptosis in a model of experimental nephropathy. Nephrol Dial Transplant 2001; 16:32-8

12. Sato H, Goto W, Yamamura J, Kurokawa M, Kageyama S, Takahara T. Therapeutic basis of glycyrrhizin on chronic hepatitis B. Antiviral Res 1996; 30:171-7.

13. Kim DH, Hong SW, Kim BT, Bae EA, Park HY, Han MJ. Biotransformation of glycyrrhizin by human intestinal bacteria and its relation to biological activities. Arch Pharm Res 2000; 23:172-7.

14. Toshiaki Makino, Nobuhiro Ohtake, Akito Watanabe, Naoko Tsuchiya, Sachiko Imamura, Seiichi Iizuka. Down-regulation of a hepatic transporter Mrp2 is involved in alteration of pharmacokinetics of glycyrrhizin and its metabolites in a rat model of chronic liver injury. DMD Fast Forward. 2008; doi:10.1124/dmd.108.021089

15. Morimoto Y, Nakajima C. Pseudoaldosteronism induced by licorice derivatives in Japan. J. Trad. Med 1991; 8:1-22.
16. Cinatl J, Morgenstern B. Glycyrrhizin, an active component of liquorice roots, and replication of SARSassociated coronavirus. Lancet 2003; 361: 2045-6.

17. Homma M, Ishihara M, Qian W, Kohda Y. Effects of long term administration of Shakuyaku-kanzo-To and Shosaiko-To on serum potassium levels. Yakugaku Zasshi 2006; 126:973-8.

18. Vladimír Tesař, Tomáš Zima, Milan Jirsa, Jiřina Crkovská, Stanislav Štípek, Zdena Vernerov. Influence of losartan and enalapril on urinary excretion of 8 isoprostane in experimental nephritic syndrome. Med Sci Monit 2002; 8: BR69-74

19. Comporti M. Lipid peroxidation. Biopathological significance. Mol Aspects Med. 1993; 14:199-207.

20. Krag S, Danielsen CC, Carmeliet P. Plasminogen activator inhibitor 1 gene deficiency attenuates TGF beta 1 induced kidney disease. Kidney Int 2005; 68:2651-66.

21. Crean JKG, Finlay D, MurphyM. The role of p42/ p44 MAPK and protein kinase B in connective tissue growth factor induced extracellular matrix protein production, cell migration and actin cytoskeletal rearrangement in human mesangial cells. J Biol Chem 2002; 277: 44187-94.

22. Zhang ZG, Liu XG, Chen GP. Significance of MMP 2 and TIMP 2 mRNA expressions on glomerular cells in the development of glomerulosclerosis. Chin Med Sci J 2004; 19:84-8.

23. Ma LJ, Jha S, Ling H. Divergent effects of low versus high dose anti TGF beta antibody in puromycin aminonucleoside nephropathy in rats. Kidney Int 2004; 65:106-15.

24. Zhang C, Meng X, Zhu Z. Role of connective tissue growth factor in renal tubular epithelial myofibroblast transdifferentiation and extracellular matrix accumulation in vitro. Life Sci 2004; 75:367-79.

Correspondence to:

WeiDe Zhong, HuiChan He Guangzhou First Municipal People's Hospital, Affiliated Guangzhou Medical College, Guangzhou 510180, China

Email:wdezhong@21cn.com 